\title{
Sustaining Ambulatory Comprehensive Medication Management Practices: Perspectives from a Minnesota Pharmacist Collaborative
}

\author{
Mckaya R. Kastner, PharmD, BCPS, and Zachariah J. Merk, PharmD, BCACP
}

\begin{abstract}
INTRODUCTION: In a time of evolving health care landscapes, pharmacists who provide comprehensive medication management (CMM) need to demonstrate financial and quality value to sustain their practice. Minnesota CMM programs started providing services in 1998, before recognition or reimbursement existed, and continue practices today. This long history lends itself well to the development of best practices for sustaining CMM practices.
\end{abstract}

PROGRAM DESCRIPTION: The Health-Systems for Integrated Medication Management (HAIMM) represents 13 Minnesota health systems, including 90 pharmacists and 121 clinics across the state. Pharmacist representatives from each of the participating health systems were polled to determine commonly implemented sustainability strategies. Common trends were identified, and the group was surveyed for important considerations on implementation of each strategy.

OBSERVATIONS: Poll results of strategies for CMM practice sustainability were placed into 2 categories: fiscal and quality-based initiatives. Each strategy was discussed to determine implementation options and lessons learned from the collaborative.

IMPLICATIONS: Numerous strategies were identified to ensure CMM practice sustainability. HAIMM's programs found it best to use a mixture of fiscal and quality-based strategies to sustain their practices.

J Manag Care Spec Pharm. 2018;24(12):1264-68

Copyright $\odot 2018$, Academy of Managed Care Pharmacy. All rights reserved.

\section{What is already known about this subject}

Comprehensive medication management (CMM) billing codes are available for clinical pharmacy services in Minnesota but often do not generate enough revenue to cover the costs of the service. Pharmacists can provide CMM services to reduce total cost of care and improve quality of care for patients.

\section{What this study adds}

Fiscal and quality-based strategies were identified to support CMM practice sustainability.

Considerations regarding the implementation of sustainability strategies were reviewed.
C omprehensive medication management (CMM) services have been offered in the state of Minnesota since 1998, for for Medicare \& Medicaid Services (CMS) and commercial payers were instituted. It was another 5 years before medication therapy management (MTM) services were officially recognized in the Medicare Prescription Drug, Improvement, and Modernization Act of 2003. ' Since being recognized, payment models for CMM have continued to improve over time. In 2006, Minnesota Medicaid started offering reimbursement for CMM Current Procedural Terminology (CPT) codes, which allowed pharmacists to bill for services provided. ${ }^{2}$ While commercial payer coverage has slowly evolved over time, it is still far from providing universal coverage of CMM services. Pharmacists also find it difficult to reimburse CMM through Medicare because pharmacists are not recognized as providers by federal CMS guidelines. With varying reimbursement rates and coverage, it can be challenging to support CMM practices on a fee-for-service structure alone.

Despite reimbursement challenges, pharmacists have demonstrated that CMM services improve patient outcomes and provide a positive return on investment through decreased total cost of care. Minnesota CMM programs have been able to demonstrate up to a $12: 1$ return on investment in overall health care costs. ${ }^{3}$ In recent years, the introduction of accountable care organizations (ACO) has provided new opportunities for CMM to demonstrate its contribution to quality care. The evolving health care landscape requires clinical pharmacists to demonstrate value in fee-for-service and value-based payment models in order to continue providing CMM.

While documents have been published on how to develop a CMM practice, literature pertaining to practice sustainability is less prevalent. ${ }^{4,5}$ This article is a collection of the experiences that have led to sustainable CMM practices in Minnesota.

\section{Program Description}

The Health-Systems for Integrated Medication Management (HAIMM) collaborative was established in 2011 and consists of 13 Minnesota health systems across the state of Minnesota. HAIMM represents over 90 pharmacists who provide services in 121 clinics. In 2016, pharmacists represented by HAIMM cared for approximately 26,000 unique patients.

HAIMM supports collaboration between health systems to advance CMM practice; promote access to CMM services; 
develop and demonstrate quality standards in practice; and disseminate findings, efforts, and experiences. HAIMM has adopted the Patient-Centered Primary Care Collaborative model of CMM for continuity of practice. ${ }^{6}$

In keeping with the goals of HAIMM, the collaborative identified a need to review current strategies in practice sustainability and share these best practices. Pharmacist representatives from each of the participating health systems were polled to determine commonly implemented sustainability strategies. These strategies were then categorized into fiscal and qualitybased initiatives. Once common trends were identified, the group discussed important considerations of implementing each strategy.

\section{Observations}

\section{Fiscal Strategies}

Optimizing CMM Billing Opportunities to Improve Revenue. HAIMM recognized 4 primary groups of payers for CMM: Medicaid, Medicare, commercial payers, and private payers. In Minnesota, Medicaid and some commercial payers allow for billing using the MTM CPT codes 99605 (initial encounter, first 15 minutes); 99606 (established encounter, first 15 minutes); and 99607 (each additional 15 minutes). ${ }^{2}$ These codes are typically the best rate of reimbursement for members of HAIMM.

For Minnesota pharmacists, Medicare only allows the lowest rate of "incident-to" billing with CPT code $99211 .^{7}$ This practice is set by Medicare Administrative Contractors (MACs), which are multistate, regional contractors responsible for administering Medicare claims. ${ }^{8}$ At the current rate of reimbursement, some health systems choose not to bill "incident-to" charges because they may not cover the administrative costs of submitting the charges. However, in certain regions of the United States, MACs have allowed for higher "incident-to" charges to be reimbursed for pharmacists, making billing "incident-to" a viable practice. ${ }^{9}$ Commercial payers' reimbursement rates and coverage of CMM services varies and is often negotiable. HAIMM felt it was important for practices to understand the different reimbursement options available in their area.

In addition to the standard payment models, health systems can negotiate for coverage of CMM services for employees or specific populations with contracted insurance providers. One of HAIMM's health systems has been able to negotiate unique contracts with payers, allowing it to bill for telephonic visits, which otherwise would not be a covered service. Recent advances in the ways that patients communicate with providers, such as direct-to-provider messaging and virtual visits, may present new opportunities for billing. Chronic care management and transitional care management represent additional opportunities for revenue but may be more successful with broad health system support. HAIMM recognized the importance of continued conversations with each of the previously mentioned payer groups to support continued reimbursement for CMM at appropriate rates.

Reduction in Total Cost of Care. CMM is well suited to support health system initiatives in reducing total cost of care. The top $1 \%$ of spenders account for $>20 \%$ of health care spending, and medication therapy problems are estimated to cost $\$ 177.4$ billion annually due mostly to hospital and long-term care readmissions. ${ }^{10,11}$ Studies have been published displaying up to a 12:1 return on investment for CMM services, as well as decreased readmissions. ${ }^{3,12}$ While there is no single recommended criterion for which patients should receive CMM services, targeting high-risk patients has been a good starting place for HAIMM members. For instance, Fairview Health System uses an internal algorithm that categorizes patients into risk categories for readmission based on age, length of stay, location, acuity, admitting diagnosis, cancer treatment, birth, and 6-month hospital history. They were able to show a $20 \%$ reduction in 30-day readmission rates in higher risk cohorts who received CMM. ${ }^{13}$ Opportunities exist to work with health system leadership to identify interventions for high-risk patients that align with system initiatives and shared savings contracts. Good documentation of outcomes before and after CMM intervention is critical in order to show direct and indirect cost savings as a result of interventions by pharmacists.

\section{Quality-Based Strategies}

Aligning with Quality Measurements. Programs identified that align with clinic, state, and national quality metrics help support continued need for their services. By reviewing individual physician and clinic quality metrics, pharmacists are able to best concentrate their efforts in areas of greatest need and potential impact. Examples of measures referenced by the group include the Merit-Based Incentive Payment System quality measures, Minnesota Community Measures, Healthcare Effectiveness Data and Information Set measures, and the Pharmacy Quality Alliance measures. Fairview Health System was able to target diabetes-related Minnesota Community Measures and showed that compared with pre-CMM numbers, patients who received $\mathrm{CMM}$ had a significantly higher rate of being optimally managed $(21.49 \%$ vs. $45.45 \%, P<0.01) .{ }^{14}$ HAIMM indicated that the ability to track and report direct pharmacist effect on quality metrics can be difficult without technology resources but that it is important to start tracking from the start of the practice.

Patient Satisfaction. In supporting pursuit of the quadruple aim framework (i.e., improving population health, enhancing patient experience, reducing cost, and improving work life of clinicians), HAIMM felt that showing the effect of CMM services on the patient experience was an integral part of quality data collection. ${ }^{15}$ A variety of patient experience surveys have been implemented by practices, including Consumer 
Assessment of Healthcare Providers and Systems (CAHPS) and Gallup surveys. In 2016, HAIMM developed a validated patient satisfaction survey specific to CMM services. ${ }^{16}$ The HAIMM survey allows the collaborative to uniformly aggregate data across its membership to further support CMM practices. Data collected from these surveys can be used to improve the patient experience during CMM visits and demonstrate support of health system patient experience goals. Showing alignment with health system initiatives has allowed programs to hire additional pharmacists and grow practices. Considerations for implementing patient satisfaction surveys include the resources and labor required to administer and collect the data. Some programs found that incorporating interns and/or residents into the survey dissemination and collection process made it a feasible endeavor.

Provider and Team-Based Care Satisfaction. In addition to information on patient satisfaction, HAIMM practices found the support of providers and care team staff to be invaluable to practice sustainability. Collecting provider opinion surveys and anecdotal experiences gave CMM practices more information about the pharmacist's effect on clinic workflow, provider satisfaction, provider workload, and areas where providers would like to see more pharmacist involvement. Hennepin Health, Park Nicollet, and North Memorial health systems have used provider/care team support and satisfaction surveys in order to support their current CMM practices. HAIMM stressed the importance of making the surveys short and accessible to providers and the power of pharmacists personally inviting providers to offer their feedback.

Providing Services that Cannot be Replicated. Pharmacists offer a unique set of skills, and programs found it helpful to identify services that are strengths of pharmacists. In addition to patient visits, many programs offer curbside consults, answer drug information questions, provide medication newsletters and present drug updates at provider meetings. By offering these services, many pharmacists are invited to committees as the drug expert and recognized for the value they bring to the team. HAIMM found it valuable to understand the various clinical activities completed by pharmacists who were frequently used by their providers to improve pharmacist use across all clinic sites.

Increasing Physician Access. Pharmacists may be able to demonstrate value through improved access to other providers. After seeing the success of improved wait times through the use of pharmacists at the Madison Veterans Affairs clinics, HealthEast Health System tracked reductions in physician third-next-available appointments to see if CMM could lead to improvements in provider access. ${ }^{17}$ However, using CMM to open up physician schedules needs to be done carefully. In cases where pharmacists are seeing patients instead of physicians, this can decrease relative value units (RVUs) for less busy providers. Alternatively, completing pre- or postwork in conjunction with a provider may increase their RVUs by allowing them to see more patients. Some of HAIMM's CMM programs are attempting to capture higher revenue through the use of transition of care management codes but have found this difficult to coordinate without broader health system support.

Tracking CMM Interventions. Tracking medication therapy problems (MTP) is a common practice in Minnesota and is likely to become the standard for measuring the interventions that result from CMM. MTPs are categorized as a problem with indication, effectiveness, safety, or adherence. Many programs within HAIMM follow the framework of MTP classification released by the Pharmacy Quality Alliance in its August 2017 consensus document. ${ }^{18}$ Tracking MTPs is one of the easiest ways to show an impact early in program development and can be especially useful in discussions with leadership. HAIMM has found it helpful to show the percentage of disease-specific MTPs found and resolved as they relate to the clinical and financial goals of a health system. Several of our practices have used the estimated benefits from these resolutions for program support and expansion. Another advantage of sharing MTP results with leadership is that they help explain to nonpharmacy staff the unique way in which pharmacists assess drugs during a CMM. For example, if a clinic system was interested in diabetes and heart failure management, a CMM practice could show that, of the MTPs tracked and resolved, 35\% involved diabetes-related medications and 20\% involved heart failure-related medications, which would align with clinic system goals.

Hospital Readmission and Care Transitions Work. The effect of CMM on hospital readmissions can vary depending on the readmission risk of the patient who receives the service. A recent study demonstrated a statistically significant lower rate of 30-day readmissions in the highest risk groups who received CMM (Average: $7.1 \%$ vs. 9.5\%, $P=0.025$; Elevated: 9.9\% vs. $21.4 \%, P<0.001$; High: $18.3 \%$ vs. $35.9 \%, P<0.001$; and Extreme: $36.4 \%$ vs. $77.7 \%, P=0.006) .{ }^{13}$ Allina Health System has been able to support CMM pharmacists as part of its ACO incentivized care management team. Internal review showed a decreased readmission rate in attributed high-risk patients who completed a CMM visit during their enrollment in care management services compared with those who declined.

Providing Service to Unique Target Populations. Consideration should be given to targeting patient populations to which payers are directing their care management efforts. Partnering with a community pharmacy and using claims data can be helpful to drive referrals based on adherence. Fairview Health System partnered with 30 of its retail sites to identify patients who could benefit from CMM, resulting in over 500 referrals in 2017. Targeting health system employees and plan members can generate value by selecting the most cost-effective 
medications. Again, HAIMM felt it was best to determine target populations with health system initiatives in mind and use informatics to identify populations.

\section{Implications}

The findings presented here were gathered from 13 health systems that spanned urban and rural settings, large and small organizations, and new and long-established CMM programs. It is recognized that members from the long-established CMM practices contributed more often to the information collected, which could be seen as a limitation to variety of responses gathered. Although HAIMM represents 90 pharmacists and 121 clinics, it is recognized that this does not include medication management practices that may be implemented in retail and insurance settings across the state. This collective also represents those most intimately involved in health system CMM programs, providing expert insight into the opportunities and challenges that exist for the sustainability of these programs.

It is also recognized that information gathered solely from Minnesota pharmacists may limit full application to outside states because of variable payer landscapes. The information discussed was largely a subjective response from HAIMM representatives and may not represent the perspectives of healthsystem leadership.

HAIMM realizes that financial pressures on health care are likely to increase in the coming years and that pharmacy services represent a significant investment for health systems. As such, it would be beneficial for pharmacists to continue to proactively work to show the value of CMM and partner with payers for reimbursement. Expanding coverage in ways that are similar to current payment models will help reduce the variation in requirements by payers and help improve adoption of coverage. Pharmacists also need to continue to improve patient and provider awareness and appreciation to drive demand for the service. Provider status may significantly affect the overall landscape of CMM reimbursement. This factor could lead to improved reimbursement rates but possible RVU expectations by health systems.

HAIMM recommends that CMM pharmacists be prepared to react to coming advancements in patient care, technology, and payment models. Expanding fields of pharmacogenomics and specialty pharmacy are well suited for CMM pharmacist involvement. Virtual access to health care providers is increasing in popularity and could prove to be beneficial for maintaining CMM services. Integration of Systemized Nomenclature of Medicine-Clinical Terminology (SNOMED CT) provides standard language for pharmacist documentation across all electronic medical records. This standardization could bring about better comparison of data, uniformity across CMM programs, and forward movement towards value-based care.

Because no one can predict how practice will change for CMM pharmacists in coming years, HAIMM considers it important to implement a variety of fiscal and value-based sustainability practices. HAIMM feels that collective use of the strategies outlined here demonstrate developed best practices for CMM sustainability in the Minnesota health system environment.

\section{Authors}

MCKAYA R. KASTNER, PharmD, BCPS, Allina Health, Richfield, Minnesota, and ZACHARIAH J. MERK, PharmD, BCACP,

HealthEast Care System, St. Paul, Minnesota.

AUTHOR CORRESPONDENCE: Mckaya R. Kastner, PharmD, BCPS, Pharmacist Practitioner, Allina Health, 407 66th St., Richfield, MN 55423. Tel.: 612.775.5050;

E-mail: mckaya.kastner@allina.com.

\section{DISCLOSURES}

No outside funding supported this study. The authors have no conflicts of interest to declare. The information within this article should not be construed as legal advice, and the Health-Systems Alliance for Integrated Medication Management (HAIMM) disclaims all liability of action or lack of action resulting from use of this content. Readers should obtain legal and/or professional advice from a licensed professional within their state.

\section{ACKNOWLEDGMENTS}

The authors acknowledge the HAIMM members for contributions to design concept, data collection, and review of the manuscript.

\section{REFERENCES}

1. Medicare Prescription Drug, Improvement, and Modernization Act of 2003. Pub L No. 108-173, 117 Stat 2066. December 8, 2003. Available at: https://www.congress.gov/108/plaws/publ173/PLAW-108publ173.pdf. Accessed October 2, 2018.

2. Minnesota Department of Human Services. Medication Therapy Management Services (MTMS). March 8, 2018. Available at: http://www.dhs. $\mathrm{mn}$.gov/main/idcplg?IdcService=GET_DYNAMIC_CONVERSION\&Revisi onSelectionMethod=LatestReleased\&dDocName=dhs16_136889. Accessed October 2, 2018.

3. Brummel A, Lustig A, Westrich K, et al. Best practices: improving patient outcomes and costs in an ACO through comprehensive medication therapy management. J Manag Care Spec Pharm. 2014;20(12):1152-58. Available at: http://www.jmcp.org/doi/abs/10.18553/jmcp.2014.20.12.1152.

4. Kliethermes MA, Brown, TR. Building a Successful Ambulatory Care Practice: A Complete Guide for Pharmacists. Bethesda, MD: American Society of HealthSystem Pharmacists; 2012.

5. American Pharmacists Association. Getting your MTM business started. 2018. Available at: http://www.pharmacist.com/getting-your-mtm-businessstarted. Accessed September 20, 2018.

6. Patient-Centered Primary Care Collaborative. The patient-centered medical home: integrating comprehensive medication management to optimize patient outcomes resource guide. $2 \mathrm{~d}$ ed. June 2012. Available at: https:// www.pcpcc.org/sites/default/files/media/medmanagement.pdf. Accessed September 20, 2018. 
7. Coffey J. Pharmacist billing for ambulatory pharmacy patient care services in a physician-based clinic and other non-hospital-based environments - FAQ. American Society of Health-System Pharmacists. May 2014. Available at: https://www.ashp.org/-/media/assets/ambulatorycare-practitioner/docs/sacp-pharmacist-billing-for-ambulatory-pharmacypatient-care-services.pdf. Accessed September 20, 2018.

8. Centers for Medicare \& Medicaid Services. What is a MAC? October 26, 2017. Available at: https://www.cms.gov/Medicare/Medicare-Contracting/ Medicare-Administrative-Contractors/What-is-a-MAC.html. Accessed September 20, 2018.

9. Hazlet TK, Karwaki TE, Downing DF. Pathway to pharmacist medical provider status in Washington State. J Am Pharm Assoc (2003). 2017;57(1):116-19.

10. Schoenman J, Chockley N. The concentration of health care spending. NIHCM Foundation Data Brief. July 2012. Available at: http://www.nihcm org/pdf/DataBrief3\%20Final.pdf. Accessed September 20, 2018.

11. Ernst FR, Grizzle AJ. Drug-related morbidity and mortality: updating the cost-of-illness model. J Am Pharm Assoc (Wash). 2001;41(2):192-99.

12. Centers for Medicare \& Medicaid Services, Center for Medicare and Medicaid Innovation. Evidence supporting enhanced medication therapy management. February 13, 2017. Available at: https://innovation.cms.gov/ Files/x/mtm-evidencebase.pdf. Accessed September 20, 2018.
13. Budlong H, Brummel A, Rhodes A, Nici H. Impact of comprehensive medication management on hospital readmission rates. Popul Health Manag. February 13, 2018 [Epub ahead of print].

14. Brummel AR, Soliman AM, Carlson AM, Oliveira DRD. Optimal diabetes care outcomes following face-to-face medication therapy management services. Popul Health Manag. 2013;16(1):28-34.

15. Bodenheimer T, Sinsky C. From triple to quadruple aim: care of the patient requires care of the provider. Ann Fam Med. 2014;12(6):573-76. Available at: http://www.annfammed.org/content/12/6/573.long. Accessed September 20, 2018.

16. Moon J, Kolar C, Brummel A, Ekstrand M, Holtan H, Rehrauer D. Development and validation of a patient satisfaction survey for comprehensive medication management. J Manag Care Spec Pharm. 2016;22(1):81-86. Available at: http://www.jmcp.org/doi/pdf/10.18553/jmcp.2016.22.1.81.

17. Galewitz P. VA Shifts to clinical pharmacists to help ease patients' long waits. Kaiser Health News. October 25, 2016. Available at: https://khn.org/ news/va-treats-patients-impatience-with-clinical-pharmacists/. Accessed September 20, 2018.

18. Pharmacy Quality Alliance. Medication therapy problem categories framework for PQA measures. August 2017. Available at: https://pqa.memberclicks.net/assets/Measures/PQA\%20MTP\%20Categories\%20Framework. pdf. Accessed September 20, 2018. 